\title{
Personalidade: estudo comparativo entre dois instrumentos de avaliação
}

\author{
Personality: Comparative study between \\ two assessment instruments
}

Tatiana de Cássia NAKANO ${ }^{1}$

\begin{abstract}
Resumo
Com o objetivo de verificar convergências entre dois modelos de avaliação da personalidade, a Escala Fatorial de Socialização e o Inventário Fatorial de Personalidade foram aplicados em 71 participantes (43 femininos/28 masculinos) com idades entre 18 e 31 anos $(M=21,11$ anos, $D P=4,28)$, escolaridade correspondente ao Ensino Médio $(n=28)$ e Ensino Superior $(n=43)$. Os resultados demonstraram que o fator 1 da Escala Fatorial de Socialização, Amabilidade, correlacionou-se significativamente e de forma positiva com oito necessidades avaliadas pelo Inventário Fatorial de Personalidade (Assistência, Intracepção, Afago, Deferência, Afiliação, Ordem, Persistência e Mudança). Os demais fatores (Pró-Sociabilidade e Confiança) não se relacionaram significativamente com nenhuma das necessidades avaliadas pelo Inventário Fatorial de Personalidade. Diferenças significativas devido ao sexo foram encontradas em relação às necessidades de intracepção, deferência e autonomia no Inventário Fatorial de Personalidade e amabilidade na Escala Fatorial de Socialização, e nenhuma diferença foi encontrada em relação à idade. Estudos que avaliem os demais fatores do Modelo dos Cinco Grandes Fatores de Personalidade são recomendados, assim como maior amplitude etária da amostra.
\end{abstract}

Palavras-chave: Escolaridade; Identidade de gênero; Modelos dos Cinco Grandes Fatores de Personalidade.

\begin{abstract}
With the aim to verify convergence between two models of personality assessment, the Agreeableness Scale and the Factor Personality Inventory were applied in 71 participants (43 female/28 male) aged between 18 and 31 years $(M=21.11$ years, $S D=4.28)$, with high school $(n=28)$ and university grade $(n=43)$. The first factor of the Agreeableness Scale, cordiality, correlated significantly and positively with eight needs assessed by Factor Personality Inventory (Assistance, Intraception, Cuddling, Deference, Affiliation, Order, Permanence and Change). Other factors (pro-sociability and trust in people) did not correlate significantly with any of the needs assessed by the Factor Personality Inventory. Significant differences due to gender were found in relation to the needs intraception, deference and autonomy in the Factor Personality Inventory and cordiality in Agreeableness Scale, and in relation to age of participants differences no were found. Studies evaluating the remaining factors of the Big Five Personality Model are recommended, as well as greater age range of the sample.
\end{abstract}

Keywords: Academic achievement; Gender identity; Big Five Personality Model.

$\nabla \nabla \nabla$

1 Pontifícia Universidade Católica de Campinas, Centro de Ciências da Vida, Programa de Pós-Graduação em Psicologia. Av. Jonh Boyd Dunlop, s/n., Jardim Ipassurama, 13060-904, Campinas, SP, Brasil. E-mail: <tatiananakano@puc-campinas.edu.br>. 
O estudo da personalidade é um tema marcado pela existência de algumas discordâncias teóricas e metodológicas a respeito do seu conceito; apesar disso, grande número de estudos e debates mostra que o construto ocupa lugar central na pesquisa psicológica (Trentini et al., 2009), pois, desde 1950, a pesquisa sobre a mensuração da personalidade vem atingindo grandes proporções, resultando em mais de uma centena de testes de personalidade existentes internacionalmente; dentre os mais numerosos e populares estão os inventários de personalidade e as técnicas projetivas (Meehl, 2000; Pervin \& John, 2004; Piotrowski, 2000). No Brasil, levantamento demonstrou que os testes de personalidade representam a maior parcela de instrumentos disponíveis no mercado profissional (Noronha, Freitas, Sartori, \& Ottati, 2002), ainda que, na literatura nacional, sejam poucos os estudos e as pesquisas nessa área de conhecimento (Noronha, 2002).

A definição do construto aponta para padrões de comportamento, atitudes e emoções que são típicas de um determinado indivíduo, de forma que os traços ou as características de personalidade difeririam de um indivíduo para outro, mas apresentariam alguma constância em uma pessoa, em diferentes contextos, bem como alguma estabilidade com o passar do tempo (Rebollo \& Harris, 2006). Por esse motivo, pesquisas mais atuais têm buscado investigar a estabilidade dos traços de personalidade ao longo da vida, bem como a influência da idade no construto (Donnellan \& Lucas, 2008; Hampson \& Goldberg, 2006; Lucas \& Donnellan, 2009; Notfle \& Fleeson, 2010).

Diante da diversidade de teorias e instrumentos disponíveis para avaliação da personalidade, estudos que visem à comparação entre resultados obtidos por meio de diferentes instrumentos que avaliam o mesmo construto se tornam importantes, visto que, conforme apontado por Hampson e Goldberg (2006), os avanços científicos na teoria da personalidade e sua medida podem resultar em uma falta de comparabilidade entre os vários tipos de medidas e de avaliações disponíveis. Nesse sentido, o presente estudo teve como objetivo inves- pela Escala Fatorial de Socialização (EFS), baseada no Modelo dos Cinco Grandes Fatores da Personalidade, e o Inventário Fatorial de Personalidade (IFP), baseado na Teoria das Necessidades Básicas de Murray, verificando convergências e divergências entre os dois modelos de avaliação da personalidade e buscando investigar ainda a influência que as variáveis sexo e idade exercem nas dimensões que são avaliadas pelos instrumentos. Ressalte-se a relevância do problema de pesquisa abordado dadas as discussões recentes em torno da área da avaliação psicológica, desencadeadas pelo Conselho Federal de Psicologia.

Em relação ao primeiro modelo teórico, Cinco Grandes Fatores (CGF), sua importância ampara-se no fato de que, conforme apontado por Prinzie, Dekovic, Reijntjes, Stams e Belsky (2009), as pesquisas em personalidade ganharam novo ímpeto e direção a partir do estabelecimento de um consenso acerca da sua estrutura, através desse modelo. Também conhecido como Big Five, ele tem se mostrado predominante na atualidade (Cuperman \& Ickes, 2009) e o mais apropriado para descrever a estrutura da personalidade, sobretudo da personalidade adulta do ponto de vista psicométrico (Garcia, 2006).

A importância desse modelo embasa-se principalmente no fato de ele ter sido aplicado em diversas amostras, em diversas culturas e por meio de numerosas fontes de informação (incluindo autoavaliação, avaliação por pares e avaliações clínicas), tendo demonstrado sua adequação nesses diferentes usos (Costa \& Widiger, 1993; McCrae, 1993; Urquijo, 2001), independentemente do país, dos instrumentos de medição utilizados e da pessoa avaliada (Furnham \& Fudge, 2008). O modelo representa uma avaliação empírica na área da personalidade (Gosling, Rentfrow, \& Swann, 2003), visto que fornece uma descrição objetiva, consistente e replicável das dimensões da personalidade humana. Apesar de relativamente recente, ele embasou diversas pesquisas transculturais que corroboraram sua universalidade (Saldanha-Silva, Sclottfeldt, Rozenberg, Teles-Santos, \& Lelé, 2007). Esse fato pode ser confirmado, de acordo com Hutz el al. (1998), perante a constatação de que os 
principais questionários e inventários de avaliação da personalidade, desenvolvidos com base em várias teorias da personalidade (e.g. o Sixteen Personality Factor Questionnaire [16-PF], o Minnesota Multiphasic Personality Inventory [MMPI], a escala de Necessidades de Murray, o California Q-Set, as escalas de Comrey, entre outros), quando submetidos a análises fatoriais, isoladamente ou em conjunto, produzem soluções compatíveis com o modelo CGF.

Nessa perspectiva, o modelo faz uso de um modelo geral composto por dois níveis hierárquicos, o qual emprega, em um primeiro nível, cinco amplos fatores, e, em um segundo nível, várias outras características, denominadas facetas (De Young, Quilty, \& Peterson, 2007). Ainda que abranja apenas cinco fatores da personalidade e, por isso, tenha sido alvo de críticas, McCrae e John (1992) confirmam a importância desses fatores, afirmando que pelo menos alguma dessas cinco dimensões seria necessária para uma adequada descrição das diferenças individuais na personalidade.

Os cinco fatores têm sido denominados Extroversão, Socialização (também chamado por alguns autores por Amabilidade), Realização (também denominado Conscienciosidade), Neuroticismo e Abertura (Nunes, Hutz, \& Giacomoni, 2009). Atualmente existem, dentro desse modelo, cinco escalas validadas e aprovadas no Sistema de Avaliação de Testes Psicológicos (SATEPSI) do Conselho Federal de Psicologia. Três delas avaliam os fatores separadamente: a Escala Fatorial de Ajustamento Emocional/Neuroticismo (Hutz \& Nunes, 2001), a EFS (Nunes \& Hutz, 2007b) e a Escala Fatorial de Extroversão (Nunes \& Hutz, 2007a), ao passo que outras duas contemplam os cinco fatores: Revised NEO Personality Inventory (NEO-PI-R) (Flores-Mendoza, 2008) e a Bateria Fatorial de Personalidade (BFP) (Nunes, Hutz \& Nunes, 2010).

Entretanto, o segundo modelo teórico envolvido no estudo, Teoria das Necessidades Básicas de Murray, apresenta algumas diferenças conceituais com os CGF, pois considera que as pessoas têm várias necessidades, que funcionam como elementos importantes nos processos motivacionais, visto que medeiam os atos e os comportamentos ao longo de toda a vida, referindo-se à prontidão ou à facilidade de cada indivíduo em reagir de determinada forma a situações específicas (Murray, 1962). Esses motivos psicológicos seriam expressos por meio de 15 necessidades (Assistência, Intracepção, Afago, Deferência, Afiliação, Dominância, Denegação, Desempenho, Exibição, Agressão, Ordem, Persistência, Mudança, Autonomia e Heterossexualidade), avaliadas no Brasil por meio de um instrumento com bastante tradição de uso, o Inventário Fatorial de Personalidade (IFP) (Pasquali, Azevedo, \& Ghesti, 1997).

A importância de estudos comparativos entre diferentes teorias ampara-se na constatação de que, embora haja uma variedade de definições para esse construto, a avaliação da personalidade irá depender da teoria adotada pelo pesquisador, de maneira que a forma como as teorias conceituam o termo acaba por definir as principais características de cada posição teórica. Ainda que a literatura venha afirmando a importância da personalidade e sua avaliação, nota-se, ainda nos dias de hoje, uma escassez de estudos que tenham por objetivo investigar diferentes instrumentos de avaliação da personalidade (embasados em teorias diferentes) com a finalidade de apontar pontos de convergência e divergência entre eles. As pesquisas nesse sentido constituem-se, em sua maioria, de acordo com Bueno, S.M.S.S. Oliveira e Oliveira (2001), de estudos que investigam correlações dos resultados dos testes de personalidade com outras variáveis, tendo sido encontrado somente um estudo internacional a respeito da convergência entre instrumentos (Furnham, 1996), que é o objetivo do estudo presente.

\section{Método}

\section{Participantes}

A pesquisa foi desenvolvida junto a 71 participantes: 43 do sexo feminino e 28 do sexo masculino; na faixa etária entre 18 e 30 anos (média de 21,11 anos, desvio-padrão de 4,28); 28 deles $(39,4 \%)$ com escolaridade correspondente ao Ensino Médio, e 43 (60,6\%) com Ensino Superior. 


\section{Instrumentos}

A Escala Fatorial de Socialização (Nunes \& Hutz, 2007a), composta por 70 itens de autorrelato que avaliam a socialização, componente da personalidade relacionado à qualidade das relações interpessoais, respondida dentro de uma escala Likert de sete pontos.

A Escala Fatorial de Socialização é composta por três facetas, denominadas Amabilidade, PróSociabilidade e Confiança nas Pessoas (Nunes, 2007). De acordo com o manual do instrumento, Amabilidade (S1) agrupa itens que descrevem o quão atenciosas, compreensivas, agradáveis, educadas, preocupadas e empáticas as pessoas procuram ser com as demais. Pró-sociabilidade (S2) agrupa itens que descrevem comportamentos de risco, concordância ou confronto com leis e regras sociais, moralidade, auto e heteroagressividade e padrões de consumo de bebidas alcoólicas. Já Confiança nas pessoas (S3) agrupa itens que descrevem o quanto as pessoas confiam nas demais e acreditam que elas não as prejudicarão.

0 instrumento encontra-se normatizado para uso em sujeitos na faixa etária entre 14 e 64 anos e escolaridade mínima do Ensino Médio. Em estudos de buscam por evidências de validade e durante o processo de normatização, Nunes e Hutz (2007b) testaram 1100 sujeitos de várias regiões do Brasil; após análise fatorial, a escala geral indicou uma estrutura composta por três fatores, cuja consistência interna foi de 0,92 por meio do alfa de Cronbach. Analisando os dados da normatização, os autores verificaram que a variável sexo mostrou-se significativa, tendo-se adotado, por esse motivo, tabelas diferenciadas entre os sexos para avaliar esses aspectos da personalidade.

O Inventário Fatorial de Personalidade (Pasquali et al., 1997), é um inventário objetivo da personalidade, baseado na teoria das necessidades básicas de Murray, composto por 135 itens, visa a avaliar o indivíduo normal (não sendo aconselhado para uso em população clínica) em 15 necessidades ou motivos psicológicos: Assistência, Intracepção, Afago, Deferência, Afiliação, Dominância, Dene-
Persistência, Mudança, Autonomia e Heterossexualidade. Além das dimensões citadas, o teste possui uma escala de desejabilidade social (12 itens) e uma escala de mentira ou validade (8 itens), de forma que, ao todo, o teste contém 155 itens, os quais devem ser respondidos dentro de uma escala tipo Likert composta por sete pontos.

Sua validação foi realizada a partir dos dados de 4308 participantes, das cinco regiões do país (predomínio das regiões Sul e Sudeste), com idade média de 21,5 anos, sendo a maioria composta por universitários. Assim como a EFS, as tabelas também são apresentadas separadas por sexo.

\section{Procedimentos}

A presente pesquisa baseou-se no estudo desenvolvido por Trentini et al. (2009), alterando-se, entretanto, o fator do Big Five enfocado. No estudo citado, foram comparados os resultados da Escala Fatorial de Neuroticismo (EFN) e o IFP: no presente estudo, o primeiro instrumento foi substituído pela EFS.

Após esclarecimentos em relação ao objetivo da avaliação e à assinatura do termo de Consentimento Livre e Esclarecido, os instrumentos foram aplicados de forma coletiva, em uma única sessão, tendo sido alterada a ordem de resposta aos instrumentos a fim de se evitar a interferência desse fator. Os instrumentos foram corrigidos de acordo com os respectivos manuais, dando origem a pontuações brutas em cada fator/motivo avaliado, resultados que foram utilizados nas análises posteriores.

\section{Resultados e Discussão}

A primeira análise realizada teve como objetivo verificar a relação entre as necessidades medidas pelo IFP e pelos fatores da EFS, considerando-se a amostra total, cujos resultados são apresentados na Tabela 1. Pode-se visualizar que o fator 1 da EFS, denominado Amabilidade, foi o único que apresentou correlações significativas e positivas com as necessidades avaliadas pelo IFP (Assistência, Intracepção, Afago, Deferência, Afiliação, Ordem, 
Persistência e Mudança); as magnitudes mais fortes foram encontradas em relação às necessidades de assistência e afiliação. Os demais fatores da EFS, Pró-Sociabilidade e Confiança, não se relacionaram significativamente com nenhuma das necessidades avaliadas pelo IFP. A pontuação total da EFS apresentou correlação positiva somente com a necessidade de deferência.

Considerando que o objetivo deste estudo foi identificar a existência de relação entre dois instrumentos de avaliação da personalidade, os resultados demonstraram a existência de pontos de convergência entre os dois modelos, uma vez que oito das 15 necessidades avaliadas no modelo de Murray (por meio do IFP) relacionaram-se de forma significativa e positiva com a amabilidade da EFS, um dos fatores medidos pelo modelo dos CGF. Entretanto, deve-se atentar que os valores de correlação estão entre 0,24 e 0,72, resultados que sugerem a existência de relação entre as variáveis de fraca a forte, de acordo com classificação apresentada por Dancey e Reidy (2006), entretanto há alguma particularidade ou diferenciação entre elas.

Considerando-se que o fator amabilidade da EFS representa a tendência de as pessoas serem atenciosas e amáveis com as demais, o que demonstra sua preocupação com as necessidades alheias bem como a necessidade de expor seu apreço por elas e preocupação em tratá-las bem, torna-se compreensível a existência das relações apontadas entre esse fator e as necessidades de assistência (sujeito com grandes desejos e sentimentos de piedade, compaixão e ternura), intracepção (típico das pessoas que julgam os outros por suas reais ou supostas intenções, não pelos atos em si, tomando de imediato uma atitude de afeto ou rejeição), deferência (valorização de atitudes como respeito, admiração e reverência) e afiliação (desejo de dar e receber afeto, sendo caracterizada por confiança, apego e lealdade), conforme descrições encontradas nos manuais dos instrumentos (Nunes \& Hutz, 2007b; Pasquali et al., 1997). Note-se que todas essas características, independentemente do instrumento em que são avaliadas, visam ao estabelecimento de uma boa relação com os outros e uma preocupação em cuidar e agradar os demais.

Entretanto, embora possam ser descritas como pessoas que se encontram constantemente voltadas ao outro, pessoas com alto escore em amabilidade também puderam ser caracterizadas, no presente estudo, por necessidades que envolvem, também, o atendimento às suas necessidades, principalmente de afago (busca constante por apoio, proteção e compreensão), ordem (tendência em manter organização, equilíbrio e precisão), persistência (tendência a levar a cabo qualquer

Tabela 1

Correlações de Pearson entre os fatores da EFS e do IFP considerando a amostra total

\begin{tabular}{|c|c|c|c|c|}
\hline Fatores & Amabilidade & Pró-sociabilidade & Confiança & EFS total \\
\hline Assistência & $0,725^{* *}$ & $-0,045$ & 0,033 & 0,073 \\
\hline Intracepção & $0,382^{* *}$ & $-0,014$ & 0,107 & 0,093 \\
\hline Afago & $0,377^{* *}$ & 0,133 & 0,136 & 0,178 \\
\hline Deferência & $0,406^{* *}$ & 0,229 & 0,155 & $0,236^{*}$ \\
\hline Afiliação & $0,651^{* *}$ & $-0,064$ & 0,069 & 0,076 \\
\hline Dominância & 0,107 & $-0,127$ & $-0,004$ & $-0,051$ \\
\hline Denegação & 0,149 & 0,187 & 0,166 & 0,196 \\
\hline Desempenho & 0,168 & $-0,057$ & 0,010 & $-0,004$ \\
\hline Exibição & 0,121 & $-0,051$ & 0,093 & 0,040 \\
\hline Agressão & $-0,092$ & $-0,133$ & $-0,023$ & $-0,085$ \\
\hline Ordem & $0,397^{* *}$ & 0,065 & 0,109 & 0,133 \\
\hline Persistência & $0,244^{* *}$ & $-0,089$ & 0,020 & $-0,005$ \\
\hline Mudança & $0,320^{* *}$ & 0,155 & 0,129 & 0,178 \\
\hline Autonomia & 0,044 & 0,078 & 0,164 & 0,132 \\
\hline Heterossexualidade & 0,182 & $-0,082$ & 0,014 & $-0,012$ \\
\hline
\end{tabular}

Nota: ${ }^{*} p \leq 0.05 ;{ }^{* *} p \leq 0.01$.

EFS: Escala Fatorial de Socialização; IFP: Inventário Fatorial de Personalidade. 
trabalho iniciado, por mais difícil que possa parecer) e mudança (gosto por experimentar coisas novas e diferentes).

Considerando-se ainda que a pontuação total na EFS descreve o quanto as pessoas tendem a confiar nas demais, acreditando em seu lado mais positivo, pode-se verificar que, além de apresentarem preocupação e desejo de ajudar, representados por um alto nível de altruísmo, também frequentemente elas tendem mais a atender aos desejos dos outros do que a lutar pelos seus interesses, de maneira a justificar a relação encontrada entre socialização e deferência, na qual respeito, admiração e reverência caracterizam essas pessoas. Nas duas descrições, portanto, pode-se notar a preocupação com o outro, mesmo que isso implique, muitas vezes, colocar-se em segundo plano.

O que se pode verificar, após a análise dos resultados, que, embora os modelos sejam consistentes entre si, poucas são as semelhanças entre eles, devendo-se reforçar o cuidado em fazer tal afirmação visto que somente um dos cinco fatores do modelo Big Five foi considerado no estudo. Embora o modelo dos CGF tenha possibilitado "uma compreensão mais profunda do que representam esses fatores, uma comparação sistemática de diversos construtos - que são avaliados por diferentes instrumentos -, e um melhor entendimento das suas diferenças e semelhanças" (Nunes et al., 2010, p.17), estudos que investiguem os demais fatores, bem como os cinco conjuntamente, são necessários para se afirmar, com mais segurança, a convergência ou divergência entre os modelos teóricos.

Uma segunda análise conduzida visou à investigação da influência das variáveis sexo e idade nos resultados dos testes, uma vez que, independentemente da teoria da personalidade utilizada, importantes constatações vêm sendo feitas pelos pesquisadores, envolvendo estudos sobre a influência de variáveis sociodemográficas que podem interferir na intensidade da expressão dos traços de personalidade, como é o caso do sexo e da idade (Nunes, Santos, \& Galvão, 2009). Assim, a Análise Multivariada da Variância foi empregada e indicou

352 que a variável sexo exerce influência significativa no desempenho dos participantes $(F=1,971$, $p \leq 0,041$ ), ao passo que a idade e a interação entre elas não se mostraram significativas.

Uma possível explicação para a ausência de influência da idade deve-se ao fato de que, na presente amostra, a amplitude etária dos participantes foi bastante reduzida, limitando-se a uma diferença de somente 13 anos entre o participante de menor idade (18 anos) e o de maior idade (31 anos). Dessa forma, pode-se levantar a hipótese de que mudanças representativas na personalidade provavelmente não ocorram em um período de tempo curto, sendo necessária nova investigação que considere uma maior diferença etária, de forma a enriquecer as discussões que vêm sendo feitas. Convém destacar que a literatura tem apontado a predominância de uma perspectiva evolucionista, a qual ressalta que, ao longo da vida, existiria uma tendência de manutenção das características básicas de personalidade (Fierro, 2004), principalmente entre os anos compreendidos entre a adolescência e a velhice, os quais têm sido vistos como anos de estabilidade e de continuidade, sem mudanças dignas de nota. Segundo o autor, as diferenças interindividuais seriam acrescidas somente com o passar do tempo, com o acúmulo de experiência, de forma que a idade comportaria um efeito progressivo de maior heterogeneidade entre as pessoas.

Concordando com esse posicionamento, Notfle e Fleeson (2010) afirmam que mudanças de personalidade na vida adulta podem ser notadas entre a adolescência tempestuosa e o período estável da meia-idade, de forma que haveria uma variabilidade interpessoal nas diferentes idades em vez de uma tendência central. Ainda de acordo com os autores, mais especificamente em relação à socialização, nota-se um aumento constante na velhice, período durante o qual as mudanças de personalidade ocorrem de forma mais positiva. Essas mudanças incluiriam, segundo Fierro (2004), certa estabilização da capacidade intelectual, uma evolução do estilo cognitivo em direção a uma maior independência de campo, reflexão, capacidade de análise e uma maior estabilidade afetiva, se comparada à adolescência. Assim, diferenças devido à idade quando se comparam, por exemplo, jovens 
adultos e idosos (Costa \& McCrae, 2002; Trentini et al., 2009) ou faixas etárias, como no estudo de Rabello et al. (2009), são encontradas na literatura, bem como diferença significativa devido a essa variável no fator socialização (Diniz \& Zanini, 2010).

Entretanto, a constatação da existência de diferenças de sexo na personalidade vai ao encontro do que tem sido relatado na literatura científica, segundo a qual essas diferenças seriam explicadas, em partes, pela influência cultural e social, situação apontada desde os primeiros estudos sobre esse aspecto (Ferreira, 1999). Nos estudos de personalidade, a masculinidade e a feminilidade, na maioria das vezes, têm sido consideradas como características ou traços internos e permanentes da personalidade (Friedman \& Schustack, 2004), embasados nos papéis sociais atribuídos e pouco tolerados quando apresentados por indivíduos do sexo oposto ao convencionado. Nesse sentido, de forma geral, algumas generalizações vêm sendo apontadas na literatura, sendo as mulheres descritas, na maioria das vezes, como emotivas, protetoras, submissas, comunicativas, sociáveis e passivas, ao passo que os homens seriam tipicamente tidos como racionais, agressivos, dominantes, objetivos e empreendedores (Andrade, 2008).

Hofstede e McCrae (2004) salientam que essa questão torna-se confusa se considerarmos que as características de um e de outro sexo podem apresentar ampla variação em diferentes culturas: se diferenças no nível médio de traços de personalidade existem, elas podem, em princípio, ser originadas por diferenças culturais. Nesse sentido, um trabalho de meta-análise conduzido por Schmitt, Reallo, Voracek e Allik (2008) apontou que as diferenças entre os sexos estaria relacionada a três pontos: (1) o papel social que tais sexos desempenham, (2) as explicações evolutivas, nas quais as diferenças entre os sexos são consideradas como inatas e (3) erro de medida, uma vez que são usados diferentes instrumentos para medir tais diferenças de sexo, usualmente do tipo autorrelato, propensos a distorções e desejabilidade social. Assim, a fim de se investigar de forma mais aprofundada a diferença

Tabela 2

Média e desvio-padrão e nível de significância por fator da EFS e do IFP, por sexo

\begin{tabular}{|c|c|c|c|c|c|}
\hline \multirow{2}{*}{ Variável } & \multicolumn{2}{|c|}{ Feminino } & \multicolumn{2}{|c|}{ Masculino } & \multirow{2}{*}{$\begin{array}{c}\text { Nível de } \\
\text { Significância }\end{array}$} \\
\hline & M & DP & M & DP & \\
\hline \multicolumn{6}{|l|}{ Necessidades IFP } \\
\hline Assistência & 51,49 & 5,96 & 50,39 & 8,21 & 0,103 \\
\hline Intracepção & 48,72 & 6,89 & 47,54 & 10,25 & $0,019^{*}$ \\
\hline Afago & 46,05 & 8,82 & 39,89 & 10,58 & 0,414 \\
\hline Deferência & 45,56 & 7,00 & 45,00 & 9,68 & $0,057^{*}$ \\
\hline Afiliação & 54,53 & 5,50 & 55,29 & 6,37 & 0,367 \\
\hline Dominância & 32,67 & 9,79 & 38,32 & 9,07 & 0,657 \\
\hline Denegação & 38,05 & 7,71 & 33,79 & 8,75 & 0,729 \\
\hline Desempenho & 49,12 & 6,82 & 51,46 & 6,07 & 0,574 \\
\hline Exibição & 35,81 & 8,78 & 41,07 & 6,46 & 0,159 \\
\hline Agressão & 29,86 & 9,18 & 36,11 & 7,91 & 0,733 \\
\hline Ordem & 43,74 & 10,84 & 41,61 & 13,32 & 0,238 \\
\hline Persistência & 40,98 & 7,90 & 48,04 & 7,37 & 0,827 \\
\hline Mudança & 47,72 & 8,54 & 48,54 & 7,99 & 0,617 \\
\hline Autonomia & 46,51 & 7,88 & 49,86 & 5,54 & $0,009^{*}$ \\
\hline Heterossexualidade & 42,77 & 7,91 & 48,18 & 7,15 & 0,558 \\
\hline \multicolumn{6}{|l|}{ Fatores EFS } \\
\hline Amabilidade & 5,68 & 0,60 & 5,37 & 0,75 & $0,027^{*}$ \\
\hline Pró-sociabilidade & 6,06 & 3,25 & 5,09 & 2,68 & 0,617 \\
\hline Confiança & 5,27 & 2,88 & 5,41 & 4,48 & 0,736 \\
\hline EFS total & 17,02 & 5,84 & 15,87 & 7,38 & 0,880 \\
\hline
\end{tabular}

Nota: ${ }^{*} p \leq 0.05$.

M: Média; DP: Desvio-Padrão; EFS: Escala Fatorial de Socialização; IFP: Inventário Fatorial de Personalidade. 
encontrada devido ao sexo, as médias, desvios-padrão e nível de significância para cada fator avaliado no IFP e na EFS são fornecidos na Tabela 2, dividida por sexo.

Conforme pode-se verificar, diferenças significativas devido ao sexo são encontradas em relação às necessidades de intracepção, deferência e autonomia no IFS e no fator Amabilidade na EFS, sendo todas mais bem pontuadas pelo sexo feminino, com exceção da necessidade de autonomia, na qual o sexo masculino destaca-se. Em relação às primeiras características (intracepção e deferência) avaliadas pelo IFP, pode-se ver que os dados corroboram o manual do instrumento, segundo o qual, de forma geral, pessoas do sexo feminino sobressairiam-se nos desejos de ajudar os outros, de compreender sentimentos e emoções, bem como na valorização do respeito e da amizade (descrição da intracepção). Tais características também vão ao encontro da descrição da necessidade de intracepção (pessoa que se deixa conduzir por sentimentos e que julga os outros por suas reais ou supostas intenções, não pelos atos em si, tomando de imediato uma atitude de afeto ou rejeição) e com a descrição da necessidade de deferência (definida como respeito, admiração, reverência, desejo de admirar e dar suporte a um superior). O manual do IFP (Pasquali et al., 1997) também apresenta que homens manifestam maiores necessidades de dominar e de se exibir, concordando com a descrição da autonomia, mais pontuada na presente pesquisa pelo sexo masculino. Autonomia representa a necessidade de sentir-se livre, sair do confinamento, resistir à coerção e à oposição. São pessoas que não gostam de executar tarefas impostas pela autoridade, gostam de agir independentemente e livremente, seguindo seus impulsos, desafiando qualquer convenção.

Em relação aos resultados obtidos na EFS, vê-se uma predominância do fator Amabilidade no sexo feminino, cujos resultados também se mostram consistentes com os apresentados no próprio manual do instrumento, no qual a variável sexo gerou perfis diferenciados para socialização, tendo sido verificadas diferenças estatisticamente significativas

354 entre homens e mulheres, com o sexo feminino apresentando médias mais altas (Nunes \& Hutz, 2007b). Tal resultado também foi relatado por Nunes et al. (2010), em um instrumento que avalia conjuntamente os cinco fatores da personalidade, as mulheres apresentaram médias mais elevadas em todas as facetas de Socialização, corroborando os achados de Gándara, Ortiz e Tello (2006). Essa característica descreve uma pessoa que tende a ser atenciosa e amável com as demais, que demonstra sua preocupação com as necessidades alheias e tende a ser proativa para resolver os problemas dos outros, bem como para expor seu apreço por eles, características que se assemelham às descrições da intracepção e da deferência no IFP, as quais também foram mais bem pontuadas pelas mulheres, de maneira que certa consistência entre as características mostra-se presente.

Uma busca na literatura mostra que outros estudos também apontam a superioridade feminina nas medidas de socialização, podendo-se citar a pesquisa de Bartholomeu, Nunes e Machado (2008), na qual diferenças significativas entre o sexo masculino e feminino na faceta de Pró-sociabilidade e na Socialização total foram relatadas, sendo as médias maiores no sexo feminino. Tais resultados indicaram, segundo os autores, que não apenas homens e mulheres apresentam níveis variados de socialização, como também apresentam padrões de associações diferenciados, que podem ser resultado do fato de mulheres emitirem mais frequentemente comportamentos relacionados à gentileza, preocupação com o próximo, maior confiança nas pessoas e menor apresentação de comportamentos agressivos, se comparadas aos homens, o que coincide com as descrições das características significativas encontradas na presente pesquisa e na pesquisa de Nunes et al. (2009), cujos resultados apontaram diferenças estatisticamente significativas nos fatores amabilidade, pró-sociabilidade e no fator geral de socialização, tendo as mulheres obtido média mais alta.

Diante da constatação de que diversos estudos que investigaram a influência dos sexos na personalidade apontaram a existência de diferenças significativas, Saldanha-Silva et al. (2007) chamam a atenção para o fato de que, tendo em vista as comprovadas diferenças entre homens e mulheres em personalidade, faz-se necessário um tratamento 
estatístico que considere tais diferenças. Nesse sentido, pode-se ressaltar que tal prática já vem sendo adotada nos manuais dos instrumentais brasileiros utilizados na presente pesquisa, dado o fornecimento de tabelas normativas classificadas por sexo.

No entanto, também são encontrados na literatura estudos que apontam perfis diferenciados de acordo com sexo. Schmitt et al. (2008), ao revisarem as diferenças de sexo em 55 culturas, relataram que, nas respostas ao inventário do Big Five, mulheres apresentaram maiores níveis de socialização do que os homens na maioria das nações. Entretanto, sabemos que essa diferença poderá variar de acordo com o contexto, uma vez que, na pesquisa de Robinson (2009), as mulheres obtiveram médias significativamente mais altas que os homens em socialização quando envolvidas em contextos com amigos e no trabalho, mas não em contextos familiares. Esses achados sugeririam, de acordo com o autor, que as diferenças de sexo em traços de personalidade seriam produto de expectativas sociais em relação aos papéis de homem e mulher, ao invés de diferenças devido a algo inato ou genético, sendo, portanto, dependente do contexto.

Ainda sobre essa questão, outra importante reflexão pode ser feita em relação aos fatores que não diferenciaram, de forma significativa, a personalidade de homens e mulheres. Uma possível hipótese levantada refere-se à necessidade atual de adaptação dos indivíduos à vivência de várias circunstâncias que exigem uma variedade de reações e comportamentos apropriados, bem como capacidade de responder a mudanças nas demandas situacionais, habilidades exigidas dos sujeitos, independentemente do sexo (Jones, Chernovetz, \& Hansson, 1978). Tais requisitos atuariam de forma a tornar propício o desenvolvimento de traços andrógenos (indivíduos com muitos traços masculinos e femininos) e indiferenciados (pouco específicos), quadro abordado por diversos autores (Candeias, 2008; Montuori \& Purser, 1995). Essas pessoas tenderiam a reagir de forma a se adaptar a inúmeras situações, sendo capazes de cuidar de outras pessoas, de serem assertivos, de expressarem suas emoções adequadamente e, ao mesmo tempo, serem racionais e independentes quando apro- priado, conforme apontaram Friedman e Schustack (2004), assemelhando-se mais entre si do que em função do sexo. Essas pessoas deixariam de manifestar somente os traços descritores correspondentes ao seu papel sexual "adequado", fato que poderia originar essa indiferenciação entre os sexos em muitas características de personalidade. Nesse sentido, alguns estudos, cujas diferenças entre os sexos, embora presentes, não se mostraram significativas, também são relatados na literatura, de maneira a fortalecer essa percepção (Diniz \& Zanini, 2010; Santos, Sisto, \& Martins, 2003; Rabelo et al., 2009).

Este estudo buscou relacionar os resultados obtidos por uma amostra em dois instrumentos de avaliação da personalidade, de forma a tentar buscar convergências entre dois modelos teóricos. Os resultados indicaram a existência de relações significativas principalmente em relação a um dos fatores da EFS (Amabilidade), que se mostrou convergente com oito das necessidades psicológicas do IFP. Deve-se salientar, no entanto, que outros sete motivos avaliados pelo IFP não se mostraram relacionados a nenhum dos fatores avaliados pela EFS, podendo-se levantar a hipótese de que essas necessidades poderiam não estar relacionadas à socialização, e sim a algum dos outros quatro fatores do modelo CGF não avaliados.

Nesse sentido, a mesma preocupação levantada por Trentini et al. (2009), ao comparar a EFN com o IFP, leva à necessidade de cautela na interpretação dos resultados da presente pesquisa, uma vez que somente um dos cinco fatores do modelo da personalidade foi avaliado, neste caso, o fator socialização. Nesse sentido, os autores recomendam que futuras pesquisas poderiam incluir os cinco fatores "a fim de identificar o quanto da variabilidade das dimensões do IFP pode ser explicada pelos CGF. Tais resultados permitiriam verificar se as necessidades oferecem, ou não, uma descrição dos aspectos da personalidade que complementam as indicações dos CGF" (Trentini et al., 2009, p.213). Uma vez que o país apresenta hoje dois instrumentos que avaliam os cinco grandes fatores (BFP e NEO-PI-R), esse estudo é incentivado.

Diferenças significativas devido ao sexo foram encontradas somente em relação às neces- 
sidades de Intracepção, Deferência e Autonomia no IFS e Amabilidade na EFS; as demais necessidades, dado o fato de não se mostrarem influenciadas por essa variável, apontaram para o fato de, na presente amostra, não se constituírem características de um sexo específico, estando presente tanto nos indivíduos do sexo feminino como do masculino. Nenhuma influência foi encontrada em relação à idade dos participantes, de forma a demonstrar que essa variável parece não influenciar a sua personalidade, embora ressalvas já tenham sido feitas em relação a essa análise, dada a pequena amplitude da faixa etária considerada no estudo.

Estudos que envolvam maior número de participantes e ampliação da faixa etária da amostra poderão apontar, de forma mais precisa, a influência que as variáveis sexo e idade exercem na personalidade, bem como a relação entre diversos outros modelos teóricos de avaliação da personalidade; no caso específico dos CGF, estudos que investiguem os fatores de realização, neuroticismo e abertura ainda não foram conduzidos e devem ser incentivados, assim como a investigação dos cinco fatores em conjunto.

\section{Referências}

Andrade, J. M. (2008). Evidências de validade do inventário dos cinco grandes fatores de personalidade para o Brasil (Tese de doutorado não-publicada). Universidade de Brasília.

Bartholomeu, D., Nunes, C. H. S. S., \& Machado, A. A. (2008). Traços de personalidade e habilidades sociais em universitários. Psico-USF, 13(1), 41-50.

Bueno, J. M. H., Oliveira, S. M. S. S., \& Oliveira, J. C. S. (2001). Um estudo correlacional entre habilidades sociais e traços de personalidade com universitários. Psico-USF, 6(1), 31-38.

Candeias, A. A. (2008). Criatividade: perspectiva integrativa sobre o conceito e sua avaliação. In M. F. Morais, \& S. Bahia (Orgs.), Criatividade: conceito, necessidades e intervenção (pp.41-64). Braga: Psiquilíbrios.

Costa, P. T., \& McCrae, R. R. (2002). NEO five-factor inventory: Interpretive report. Lutz: Psychological Assessment Resources.

Costa, P. T., Jr., \& Widiger, T. A. (1993). Introduction. In P. T. Costa, \& T. A. Widiger (Eds.), Personality disorders and the five-factor model of personality (pp.1-10). Washington: American Psychological Association.

Cuperman, R., \& Ickes, W. (2009). Big five predictors of 356 behavior and perceptions in initial dyadic interactions:
Personality similarity helps extraverts and introverts, but hurts "disagreeables". Journal of Personality and Social Psychology, 97(4), 667-684.

Dancey, C. P., \& Reidy, J. (2006). Estatística sem matemática para psicologia. Porto Alegre: Artmed.

De Young, C. G., Quilty, L. C., \& Peterson, J. B. (2007). Between facets and domains: 10 aspects of the big five. Journal of Personality and Social Psychology, 93(5), 880-896.

Diniz, S. S., \& Zanini, D. S. (2010). Relação entre fatores de personalidade e estratégias de coping em adolescentes. Psico-USF, 15(1), 71-80.

Donnellan, M. B., \& Lucas, R. E. (2008). Age differences in the big five across the life span: Evidence from two national samples. Psychology and Aging, 23(3), 558-566.

Ferreira, M. C. (1999). Identidade de gênero e atitudes sobre a mulher. Psicologia: Teoria e Pesquisa, 15(3), 249-255.

Fierro, A. (2004). O desenvolvimento da personalidade na idade adulta e na velhice. In C. Coll, A. Marchesi, \& J. Palácios. Desenvolvimento psicológico e educação (Vol.1, pp.404-420). Porto Alegre: Artmed.

Flores-Mendoza, C. (2008). Inventário de Personalidade NEO Revisado: NEO PI-R-Manual. São Paulo: Vetor.

Friedman, H. S., \& Schustack, M. W. (2004). Teorias da personalidade: da teoria clássica à pesquisa moderna. São Paulo: Prentice Hall.

Furnham, A. (1996). The big five versus the big four: The relationship between the Myers-Briggs Type Indicator (MBTI) and NEO-PI Five Factor Model of Personality. Personality and Individual Differences, 21(2), 303-307.

Furnham, A., \& Fudge, C. (2008). The big five model of personality and sales performance. Journal of Individual Differences, 29(1), 11-16.

Gándara, M. V. B., Ortiz, M. A. C., \& Tello, F. P. H. (2006). Análisis transversal de los cinco factores de personalidad por sexo y edad en niños españoles. Revista Latinoamericana de Psicología, 38(3), 567-577.

Garcia, L. F. (2006). Teorias psicométricas da personalidade. In C. E. Flores-Mendoza, \& R. Colom (Orgs.), Introdução à psicologia das diferenças individuais (pp.219-242). Porto Alegre: Artmed.

Gosling, S. D., Rentfrow, P. J., \& Swann, W. B. Jr. (2003). A very brief measure of the big five personality domains. Journal of Research in Personality, 37(6), 504-528.

Hampson, S. E., \& Goldberg, L. R. (2006). A first large cohort study of personality trait stability over the 40 years between elementary school and midlife. Journal of Personality and Social Psychology, 91(4), 763-779.

Hofstede, G., \& McCrae, R. R. (2004). Personality and culture revisited: Linking traits and dimensions of culture. Cross-Cultural Research, 38(1), 52-88.

Hutz, C. S., Nunes, C. H. S., Silveira, A. D., Serra, S. J., Anton, M., \& Wieczorek, L. S. (1998). O desenvol- 
vimento de marcadores para a avaliação da personalidade no modelo dos cinco grandes fatores. Psicologia Reflexão e Critica, 11(2), 395-411.

Hutz, C. S., \& Nunes, C. H. S. S. (2001). Escala fatorial de neuroticismo. São Paulo: Casa do Psicólogo.

Jones, W. H., Chernovetz, M. E. O., \& Hansson, R. O. (1978). The enigma of androgeny: Differential implications for males and females? Journal of Consulting and Clinical Psychology, 46(2), 298-313.

Lucas, R. E., \& Donnellan, M. B. (2009). Age differences in personality: Evidence from a nationally representative Australian sample. Developmental Psychology, 45(5), 1353-1363.

McCrae, R. R. (1993). Opennes to experience as a basic dimension of personality. Imagination, Cognition and Personality, 13(1), 39-55.

McCrae, R. R., \& John, O. (1992). An introduction to the five-factor model and its applications. Journal of Personality, 60(2), 174-214.

Meehl, P. E. (2000). The dynamics of "structured" personality tests. Journal of Clinical Psychology, 56(3), 367-373.

Montuori, A., \& Purser, R. (1995). Deconstructing the lone genius myth: Towards a socio-ecological view of creativity. Journal of Humanistic Psychology, 35(3), 69-112.

Murray, H. (1962). Explorations in personality. New York: Science Editions.

Notfle, E. E., \& Fleeson, W. (2010). Age differences in big five behavior averages and variabilities across the adult life span: Moving beyond retrospective, global summary accounts of personality. Psychology and Aging, 25(1), 95-107.

Noronha, A. P. P. (2002). Análise de testes de personalidade: qualidade do material, das instruções, da documentação e dos itens qualidade de testes de personalidade. Estudos de Psicologia (Campinas), 19(3), 55-65.

Noronha, A. P. P., Freitas, F. A., Sartori, F. A., \& Ottati, F. (2002). Informação contidas nos manuais de testes de personalidade. Psicologia em Estudo, 7(1), 143-149.

Nunes, C. H. S. (2007). Lançamento da Escala Fatorial de Extroversão (EFEX) e Escala Fatorial de Socialização (EFS). Avaliação Psicológica, 6(1), 103-106.

Nunes, C. H. S., \& Hutz, C. S. (2007a). Escala Fatorial de Extroversão: manual técnico. São Paulo: Casa do Psicólogo.

Nunes, C. H. S., \& Hutz, C. S. (2007b). Escala Fatorial de Socialização: manual técnico. São Paulo: Casa do Psicólogo.

Nunes, C. H. S., Hutz, C. S., \& Giacomoni, C. H. (2009). Associação entre bem estar subjetivo e personalidade no modelo dos cinco grandes fatores. Avaliação Psicológica, 8(1), 99-108.

Nunes, C. H. S. S., Hutz, C. S., \& Nunes, M. F. O. (2010). Bateria fatorial de personalidade: manual técnico. São Paulo: Casa do Psicólogo.
Nunes, M. F. O., Santos, A. A., \& Galvão, N. B. S. (2009). Extroversão e socialização em estudantes de psicologia e engenharia. Revista Brasileira de Orientação Profissional, 10(2), 69-79.

Pasquali, L., Azevedo, M. M., \& Ghesti, I. (1997). Inventário fatorial de personalidade: manual técnico e de avaliação. São Paulo: Casa do Psicólogo.

Pervin, L. A., \& Jonh, O. P. (2004). Personalidade: teoria e pesquisa ( $8^{\mathrm{a}} \mathrm{ed}$.) Porto Alegre: Artmed.

Piotrowski, C. (2000). How popular is the personality assessment inventory in practices and training? Psychological Reports, 86(1), 65-66.

Prinzie, P., Dekovic, M., Reijntjes, A. H. A. Stams, G. J. M., \& Belsky, J. (2009). The relations between parent's big five factors and parenting: A meta-analytic review. Journal of Personality and Social Psychology, 97(2), 351-362.

Rabelo, I. S., Leme, I. F. A. S., Rossetti, M. O., Pacanaro, S. V., Guntert, I. B., \& Barrionuevo, V. L. (2009). Aplicação das escalas de personalidade EFS e EFEx no contexto organizacional. Avaliação Psicológica, 8(1), 109-117.

Rebollo, I., \& Harris, J. R. (2006). Genes, ambiente e personalidade. In C. E. Flores-Mendoza, \& R. Colom (Orgs.), Introdução à psicologia das diferenças individuais (pp.300-322). Porto Alegre: Artmed.

Robinson, O. C. (2009). Variability and consistency in big 5 trait expression across three interpersonal contexts. Journal of Individual Differences, 30(4), 201-208.

Saldanha-Silva, R. S., Schlottfeldt, G., Rozenberg, M. P., Teles-Santos, M. T., \& Lelé, A. J. (2007). Replicabilidade do modelo dos cinco grandes fatores em medidas de personalidade. Mosaico Estudos em Psicologia, 1(1), 37-49.

Santos, A. A. A., Sisto, F. F., \& Martins, R. M. M. (2003). Estilos cognitivos e personalidade: um estudo exploratório de evidências de validade. Psico-USF, 8(1), 11-19.

Schmitt, D. P., Reallo, A., Voracek, M., \& Allik, J. (2008). Why can't a man be more like a woman? Sex differences in big five personality traits across 55 cultures. Journal of Personality and Social Psychology, 94(1), 168-182.

Trentini, C. M., Hutz, C. S., Bandeira, D. R., Teixeira, M. A. P., Gonçalves, M. T. A., \& Thomazoni, A. R. (2009). Correlações entre a EFN - Escala Fatorial de Neuroticismo e o IFP - Inventário Fatorial de Personalidade. Avaliação Psicológica, 8(2), 209-217.

Urquijo, S. (2001). Modelos circumplexos da personalidade. In F. F. Sisto, E. T. B. Sbardelini, \& R. Primi (Orgs.), Contextos e questões da avaliação psicológica (pp.31-49). São Paulo: Casa do Psicólogo.

Recebido em: 29/3/2012

Aprovado em: 8/5/2012 
